\title{
DISEÑO DIDÁCTICO PARA EL DESARROLLO DE DESTREZAS BÁSICAS DE PROGRAMACIÓN POR MEDIO DEL PROGRAMA SCRATCH A ESTUDIANTES DEL GRADO QUINTO DEL COLEGIO SEMINARIO DIOCESANO DE DUITAMA
}

\author{
MSc. (C) Oscar Leonardo Briceño Guevara*, PhD. Julio Enrique Duarte**, PhD. Flavio \\ Humberto Fernández Morales*** \\ *Universidad Pedagógica y Tecnológica de Colombia, Duitama, Boyacá, Colombia. \\ E-mail:\{ lein0710, julioenriqued1, flaviofm1\}@gmail.com
}

\begin{abstract}
Resumen: En este trabajo se presenta una investigación basada en el desarrollo de destrezas básicas de programación, por medio del programa Scratch, para el mejoramiento del proceso de aprendizaje de la asignatura de Ciencias Naturales, CN. La metodología utilizada fue cuantitativa, con un grupo control y otro experimental. La población correspondió a estudiantes de grado quinto, donde se evaluaron dos grupos, cada uno de 30 estudiantes cuyas edades oscilan entre los 9 y 10 años. Luego de utilizar Scratch con los estudiantes del grupo experimental, se encontró que mejoraron su desempeño, pasando del 54\% que aprobaron la prueba diagnóstica, al $85 \%$ que aprobaron la prueba final. Además, los estudiantes del grupo control no presentaron cambios significativos en su rendimiento. Los resultados revelan que el desempeño académico de los estudiantes en $\mathrm{CN}$, puede mejorar apoyándose en actividades diseñadas por ellos mismos en Scratch, facilitando la asimilación de los conceptos de esta área.
\end{abstract}

Palabras clave: Scratch, habilidades de programación, ciencias naturales, enseñanzaaprendizaje, Educación.

\section{DIDACTIC DESIGN FOR THE BASIC PROGRAMMING SKILLS DEVELOPMENT THROUGH THE SCRATCH PROGRAM FOR STUDENTS IN THE FIFTH GRADE OF DIOCESANO SEMINARIO SCHOOL IN DUITAMA.}

\begin{abstract}
This work presents a research based on the development of basic programming skills, through the program Scratch to improve the Natural Sciences learning process. It was used a qualitative methodology, with a control and experimental group. The population under study is aimed at fifth graders, with two groups of 30 students each whose ages range between 9 and 10 years old.

After using the Scratch program with the experimental group, the results show that students improved their academic performance in Natural Sciences, going from 54\% of students who succeeded on the diagnostic test to $85 \%$ who passed the final test and showing no significant changes on the students' performance in the control group. Findings show that students' academic performance in Natural Sciences can improve by supporting this process in activities of their own devising when using Scratch facilitating their knowledge apprehension.
\end{abstract}

Keywords: Scratch, programming skills, natural sciences, teaching-learning, Education. 


\section{INTRODUCCIÓN}

Las Tecnologías de la Información y la Comunicación (TIC), son un factor de vital importancia en la nueva economía global y en los rápidos cambios que están tomando lugar en la sociedad. En la última década, las nuevas herramientas tecnológicas de la información y la comunicación han producido un cambio profundo en la manera en que los individuos se comunican e interactúan en el ámbito de los negocios (Cadena et al., 2015; Moya et al., 2019). Igualmente, han provocado cambios significativos en la industria, la agricultura, la medicina, el comercio, la ingeniería y otros campos (Criollo et al., 2014; Mejía et al., 2017; Marino et al., 2017; Giraldo et al., 2017). Las TIC también tienen el potencial de transformar la naturaleza de la educación en cuanto a dónde y cómo se produce el proceso de aprendizaje, así como de introducir cambios en el rol de profesores y alumnos (Cruz et al., 2019).

Los sistemas educativos de todo el mundo se enfrentan actualmente al desafío de utilizar las TIC, para proveer a sus alumnos con las herramientas y conocimientos necesarios para el siglo XXI (Niño et al., 2018; Galeano et al., 2018). En 1998, el Informe Mundial sobre la Educación de la UNESCO, Los docentes y la enseñanza en un mundo en mutación, describió el profundo impacto de las TICs en los métodos convencionales de enseñanza y de aprendizaje, augurando también la transformación de tal proceso y la forma en que los docentes y alumnos acceden al conocimiento y la información (Sanz y González, 2018).

Con el advenimiento de las nuevas tecnologías, el énfasis de la profesión docente está cambiando desde un enfoque centrado en el profesor y basado en clases magistrales, hacia una formación centrada principalmente en el alumno, dentro de un entorno interactivo de aprendizaje (Niño et al., 2016; Martínez y Pinto, 2018). El diseño e implementación de programas de capacitación docente que utilicen las TICs, efectivamente es un elemento clave para lograr reformas educativas profundas y de amplio alcance (Vega y Ruiz, 2018).

Las instituciones de formación docente deberán optar entre asumir un papel de liderazgo en la transformación de la educación, o bien quedar rezagadas en el camino del incesante cambio tecnológico (Jiménez et al., 2017). Para que la educación pueda explotar al máximo los beneficios de las TIC en el proceso de aprendizaje, es esencial que tanto los futuros docentes como los docentes en actividad sepan utilizar estas herramientas (Salcedo et al., 2017).

Las instituciones y los programas de formación deben liderar y servir como modelo para la capacitación, tanto de futuros docentes como de docentes en ejercicio, en lo que respecta a nuevos métodos pedagógicos y nuevas herramientas de aprendizaje (Piratoba y Rojas, 2014; Garzón y Romero, 2018). También deben tomar la iniciativa para determinar la mejor forma de utilizar las nuevas tecnologías, en el contexto de las condiciones culturales y económicas y de las necesidades educativas de cada país (Lopez et al., 2017; Chiape y Romero, 2018).

En Colombia se han adelantado proyectos como "Computadores para Educar", del Ministerio de las TIC, que llevó computadores y tablets a las diferentes instituciones de educación básica y media (García et al., 2017). Esto ha generado un reto para los docentes, en cuanto a la integración de estos dispositivos en el aula, de modo que mejoren del proceso enseñanza-aprendizaje de los estudiantes 
(Guzmán y Alvarez, 2014; Barrera et al., 2017).

En este sentido, los docentes deben actualizarse en el manejo de paquetes computacionales que les permitan optimizar su labor docente, independientemente de la disciplina que orienten (Berdugo et al., 2018; Ruiz y Duarte, 2018). Ejemplo de ello es el lenguaje de programación Scratch, que puede ser usado de forma provechosa para el desarrollo de competencias básicas de programación en niños (Moreno et al., 2015). Su gran ventaja es la interactividad y su adaptación a nuevos ambientes, lo que facilita su aplicación por usuarios poco experimentados (Resnick et al., 2009).

El uso de las nuevas tecnologías implica algo más que la rutina de engancharse a un dispositivo electrónico. Se trata de un avance donde los teléfonos inteligentes, Ipads, redes sociales y comunidades, se han convertido en parte del diario vivir, no solo de los estudiantes, sino también de los docentes (Gutiérrez, 2018; Pulido, 2018). En este sentido, se cuenta con un punto a favor en la educación, ya que hay un grado de motivación y manejo de la tecnología, lo que permite iniciar a una temprana edad, involucrando al estudiante en un proceso de autoaprendizaje con respecto al pensamiento lógico (Palma y Sarmiento, 2015).

En la actualidad, los modelos educativos innovadores deben fomentar ambientes de aprendizaje interactivos, dinámicos y asincrónicos, donde el docente se encuentre comprometido con el aprendizaje de sus alumnos y cumpla un papel como asesor y facilitador (Páez et al., 2016; Cárdenas y Martínez, 2015). En estos modelos, los alumnos se convierten en autores de cambio con habilidades y modos de trabajo innovadores en los cuales utilizan tecnologías de vanguardia, materiales didácticos, recursos de información y contenidos digitales. (Ordóñez et al., 2019; Buitrago, 2014; Avella et al., 2017). Una propuesta metodológica para utilizar estos modelos educativos son los Ambientes de Aprendizaje Mediados por las TIC, ya que el desarrollar un ambiente de este tipo no es trasladar la docencia de las aulas de clase a un ambiente virtual, sino mediarlas con las nuevas Tecnologías (Barrera et al., 2017; Riscanevo, 2016).

El Colegio Seminario Diocesano, de la ciudad de Duitama, Boyacá, posee unas instalaciones adecuadas para desarrollar este tipo de ambientes de aprendizaje, pues cuenta con salas dotadas para el uso de los estudiantes. El MEN, en su guía 30, da las pautas de cómo se rigen las competencias de la tecnología e informática. El colegio diseñó su propio plan de estudios acorde a los lineamientos presentados en ese documento; esta guía contempla un plan decenal que inició en el 2006 y culminó en el 2015. Es por esto que en este nuevo plan de estudios se involucraron las competencias en TIC desde edades tempranas, empezando en primer grado de primaria hasta el grado undécimo de bachillerato, resaltando la importancia de la solución de problemas reales.

El objetivo de este trabajo consistió en identificar las dificultades en el aprendizaje de las asignaturas impartidas en el grado quinto de primaria, con el fin de articularlas con el uso de las TIC y poder implementar diseños didácticos que medien la comprensión de las temáticas de mayor dificultad. A continuación, se presentan los resultados de utilizar el software SCRATCH en el proceso de enseñanza-aprendizaje del área de ciencias naturales, que se identificó como la más problemática en el grado quinto. Este programa estimula el pensamiento algorítmico y lógico de los estudiantes, para perder el miedo a programar y poder 
articularse con áreas de mayor dificultad y hacerlas fáciles, didácticas e interactivas.

\section{MARCO TEÓRICO Y METODOLOGÍA}

\subsection{Ambientes de aprendizaje}

El Colegio Seminario Diocesano, de la ciudad de Duitama, donde se desarrolló esta investigación, cuenta con un modelo de aprendizaje basado en el constructivismo, el cual se toma como base para el diseño didáctico propuesto en este trabajo. La idea central del constructivismo reside en que el practicante es quien construye su propio juicio de aprender, con las ayudas e información que proporcione o que encuentre en el entorno exterior. En simples palabras, puede denominarse la teoría constructivista a aquella que entiende que el conocimiento es el resultado de un proceso de construcción o reconstrucción de la realidad, que tiene su origen en la interacción entre personas y el mundo que lo rodea (Sepúlveda 2015; Jiménez y Sánchez, 2019).

Según Chaupart et al. (2014), se puede definir un Ambiente de Aprendizaje como el conjunto de entornos de interacción, sincrónica y asincrónica, donde, con base en un programa curricular, se lleva a cabo el proceso de enseñanza-aprendizaje a través de un sistema de administración del mismo. Adicionalmente, Angarita et al. (2014), señala que: "un ambiente de aprendizaje constituye un espacio propicio para que los estudiantes obtengan recursos informáticos y medios didácticos, para interactuar y realizar actividades encaminadas a metas y propósitos educativos previamente establecidos".

\subsection{Scratch}

Scratch es un lenguaje de programación visual, desarrollado por el Instituto Tecnológico de Massachusetts bajo la dirección del Dr. Mitchel Resnick (Vidal et al., 2015). Es utilizado por estudiantes, profesores y padres para crear animaciones, juegos educativos e interactivos, de forma sencilla y lúdica (Garrido, 2015). El entorno fue diseñado como medio de expresión para niños y jóvenes, permitiendo expresar sus ideas de forma creativa, desarrollando igualmente habilidades de pensamiento lógico y algorítmico (Resnick et al., 2009).

Lo más entretenido para aprender con Scratch es que no se requiere insertar códigos, solamente se necesita estar habituado con el entorno, sus herramientas y sus bloques de trabajo.

En este sentido, Scratch es "Un lenguaje de programación diseñado para niños con el objetivo de enseñarles conceptos de programación a una edad temprana, de modo que puedan desarrollar sus habilidades creativas" (Serrano, 2012). Como sugiere Papert (1980), "este lenguaje de programación debe tener un suelo bajo que significa empezar fácil a programar, y un techo alto que son oportunidades de crear proyectos más complejos con el tiempo". De aquí que todos podemos, y deberíamos aprender a programar, empezando a edades tempranas.

La capacidad de codificar permite escribir nuevos tipos de cosas: historias interactivas, juegos, animaciones y simulaciones (Resnick et al., 2009). En la figura 1, se muestra como se realiza una secuencia para la elaboración de diseños en programación para niños y jóvenes en scratch, sin importar la edad. 


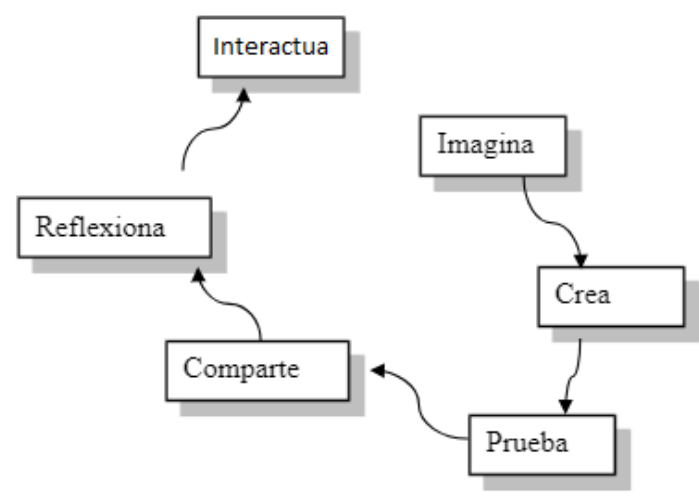

Fig. 1. Filosofía de scratch en la creación de diseños para niños y jóvenes. (Resnick et al., 2009).

\subsection{Unidad Didáctica}

La unidad didáctica implementada en este proyecto, se desarrolló en las áreas de tecnología e informática y ciencias naturales, ya que esta última fue la que los estudiantes identificaron como la de mayor dificultad. Se procede a realizar una transversalidad entre estas dos áreas, ya que se va a enseñar la temática de ciencias a través de una herramienta tecnológica. Las actividades se establecieron a partir de las competencias, contenidos, logros y estándares propuestos para el grado quinto.

De acuerdo con Escamilla (2009), "una forma de planificar el proceso de enseñanza-aprendizaje, alrededor de un elemento de contenido se convierte en eje integrador del proceso, aportándole consistencia significativa". Esta forma de organizar conocimientos y experiencias debe considerar la diversidad de elementos que contextualizan el proceso en un nivel de desarrollo del alumno.

El aprendizaje de la programación de computadoras pertenece a lo que se conoce como saber hacer o saber procedimental. El saber hacer es aquel conocimiento que se adquiere con la ejecución de procedimientos, estrategias, técnicas, habilidades, destrezas, métodos, etc. A diferencia del saber procedimental que es de tipo práctico (Pasos et al., 2015). Esto se refleja en el interés de los estudiantes, quienes se muestran deseosos de aprender más allá de lo establecido en el programa oficial del curso, y de lo enseñado por el docente.

\subsection{Diseño Metodológico.}

La investigación tiene un enfoque cuantitativo de tipo experimental, con dos grupos de trabajo: uno experimental y otro control (Zuluaga, 2017). Se busca establecer el efecto que tiene un ambiente de aprendizaje basado en Scratch, sobre el rendimiento académico en ciencias naturales de estudiantes del grado quinto de primaria.

El proyecto se desarrolló en el colegio Seminario Diocesano, de Duitama, Boyacá.

Es una entidad educativa de perfil privado, heterogéneo y de orientación religiosa, que ofrece los niveles educativos de transición, preescolar, básica y media. El establecimiento cuenta con 1250 estudiantes aproximadamente, con instalaciones apropiadas; en total 40 salones, cada uno con video beam, computador portátil, internet y aula virtual.

La población de estudio corresponde a estudiantes del grado quinto, donde se evaluaron dos grupos, cada uno cuenta con 30 estudiantes de estrato 4, y su edad oscila entre los 9 y 10 años. Cada grupo está conformado por 16 niños y 14 niñas. 
La investigación se desarrolla en cuatro fases:

i) La identificación de la temática en ciencias naturales básicas, para la creación del diseño didáctico mediado por las TIC, dirigido a los estudiantes del grado quinto.

ii) El diseño didáctico mediado por las TIC para la enseñanza de la temática en ciencias naturales en estudiantes de quinto grado, que haga uso de las herramientas existentes en SCRATCH.

iii) Aplicación del diseño didáctico mediado por las TIC, con la población objeto de estudio, para evaluar la eficiencia en el aprendizaje de las ciencias naturales y plantear ajustes.

iv) Valoración de los resultados alcanzados en la implementación.

\section{RESULTADOS Y DISCUSIÓN}

\subsection{Diseño didáctico propuesto}

El diseño didáctico se planteó a partir de una encuesta, que permitió identificar a las ciencias naturales como el área con mayor dificultad para los estudiantes de grado quinto. Luego se aplicó una prueba específica de 10 preguntas, relacionadas con las ciencias naturales. Se evaluaron los conocimientos previos relacionados con la materia, correspondientes a nociones elementales tras el paso por los grados anteriores.

Los resultados del diagnóstico indican que en el grupo 5-A, el 85\% de los estudiantes poseen conocimientos previos de la asignatura; mientras que en el grupo 5-B, el $83 \%$ desaprobaron, como se evidencia en la figura 2. En vista de lo anterior, se seleccionó a 5-B como el grupo experimental, atendiendo a las que tienen mayores falencias en los conceptos básicos de la asignatura de ciencias naturales.

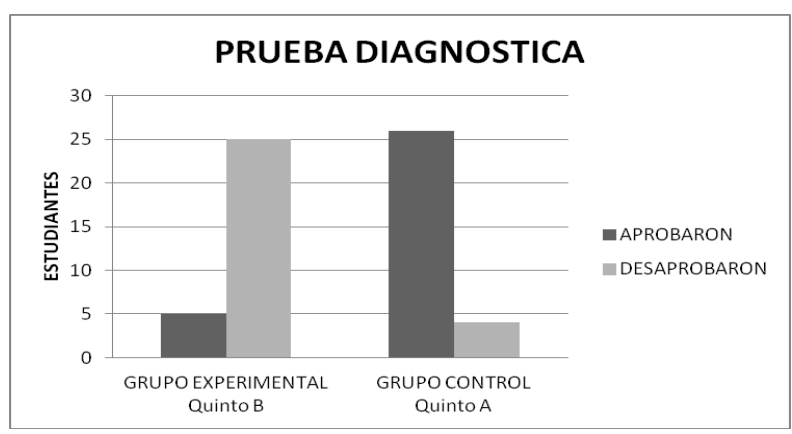

Fig.2. Evaluación de conocimientos previos básicos.

A partir del análisis de la prueba específica, donde se evidencia la falencia de conocimientos en el área de ciencias naturales, se establecieron los componentes básicos para la intervención en el aula. En este caso, los actores del proceso enseñanza-aprendizaje, son: los estudiantes del grupo 5-A como grupo control, 5-B como grupo experimental, y el docente del área de tecnología, mientras que la mediación se efectúa a través del lenguaje de programación Scratch.

El diseño didáctico se basó en el aprendizaje cooperativo, donde los estudiantes trabajaron por parejas, lo que significó mayor apoyo entre ellos.

Además, varios estudiantes se desenvolvieron con gran facilidad consultando en la red, mientras que otros realizaron el bosquejo de la programación en hojas de papel para tener más claridad a la hora de interactuar con Scratch. 


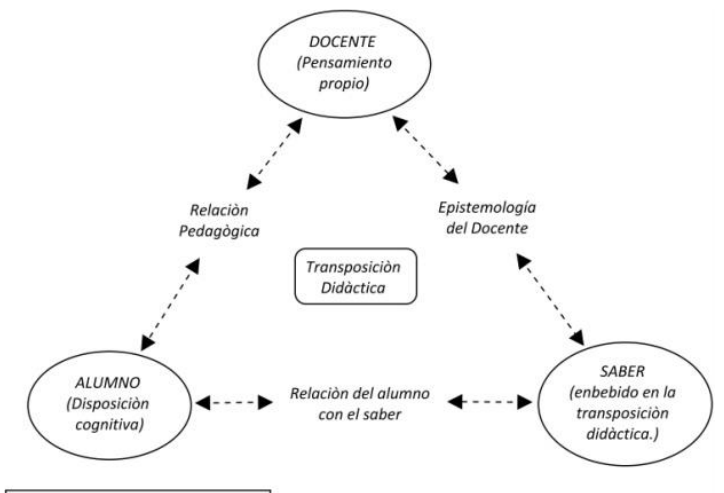

Fuente: (Chevallard y Johsua - 1982)

Fig.3. Componentes pedagógicos de la Transposición Didáctica (Chevallard y Johsua, 1982).

En esta investigación se tomaron como actividades: la visualización de videos relacionados con el lenguaje de programación Scratch, interacción con páginas web relativas al tema (scratch.mit.edu), desarrollo de programaciones sencillas, prácticas $\mathrm{y}$ textuales. Esto se complementó con material destinado específicamente al trabajo en el aula de clase (ver figura 3 ).

El objetivo de esta investigación es contribuir a mejorar las competencias básicas en ciencias naturales, aprovechando el pensamiento lógico programable, a través del software $S C R A C T H$. En la figura 4 se muestra la estructura del ambiente de aprendizaje diseñado.

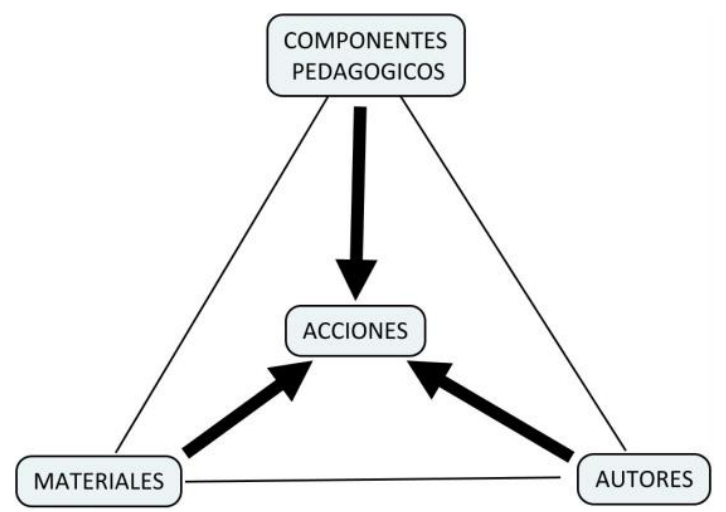

Fig.4. Ambiente de Aprendizaje Diseñado.
En la figura 4 se observa la interacción con cada uno de los actores en el ambiente de aprendizaje, donde los materiales utilizados se basan en: enlaces de páginas relacionadas en línea, videos, visualización de temas, ejercicios prácticos en el aula que complementan los saberes, conceptos entre los mismos estudiantes, así como en instrumentos creados para el desarrollo de las competencias establecidas.

El docente puede establecer el nivel de conocimiento y habilidades a ser adquiridas, a través del desarrollo de guías de aprendizaje y el proyecto final.

La apropiación de saberes en los grupos de estudiantes, afianza el momento del desarrollo de las guías de aprendizaje, trabajadas en el aula de clase. Esto se refuerza con la asesoría del docente, y el resultado de las programaciones desarrolladas en el software.

Otro objetivo implícito en el ambiente de aprendizaje, es el de contribuir al desarrollo del pensamiento algorítmico, para el progreso de aquellas asignaturas que el estudiante no logra entender, o comprender, debido al nivel de exigencia.

\subsection{Actividades Desarrolladas}

La labor a ser desarrollada se establece en varias actividades dentro del software, como se muestra en la figura 5, que corresponde al diseño didáctico para el aprendizaje del sistema óseo. 


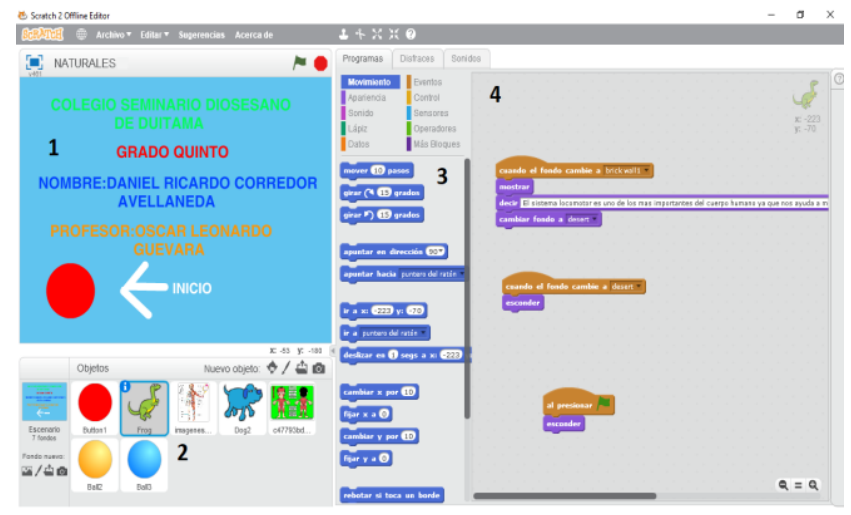

Fig.5. Actividades para el aprendizaje del sistema óseo propuestas en Scratch.

El entorno de la figura 5, está conformado por 4 interfaces para que el niño interactué y sea más llamativo cuando programe. En el número 1 se aprecia la visualización de cómo va el proceso de programación. En el número 2 se tienen los objetos que el niño va a insertar para la programación, y como son muñecos el estudiante se ve más interesado al programar. En el número 3 se encuentran los bloques y dentro de estos se localizan los comandos, los cuales el estudiante elige para los movimientos y para la programación. En el número 4 se encuentra el área de trabajo, donde el niño inserta los comandos y los une como si fueran bloques, para darle la animación a los objetos que previamente se habían insertado.

Las actividades de la figura 5 se desarrollan en el aula de clase, con ejercicios en línea, tareas de programación en documentos, procesos de aprendizaje del software y recomendaciones del docente para complementar la teoría y la programación en el salón de clase. La actividad con los estudiantes en la institución, se desarrolló en un ambiente de trabajo tranquilo y sin interrupciones como lo es el laboratorio de Informática.

Al finalizar el trabajo con el ambiente de aprendizaje, se hizo una prueba práctica donde el estudiante, con base en la programación que desarrolló, pudo evidenciar la apropiación de las temáticas del área de ciencias naturales.

En la figura 6 se observa una mejora entre las pruebas inicial y final (ver figura 2). Los estudiantes del grupo experimental mejoraron su desempeño, pasando del $54 \%$ que aprobaron inicialmente, al $85 \%$ de aprobados en la prueba final. El grupo control, luego de realizar las actividades con un ambiente de aprendizaje tradicional, no presentó cambios significativos al comparar los resultados de las pruebas inicial y final.

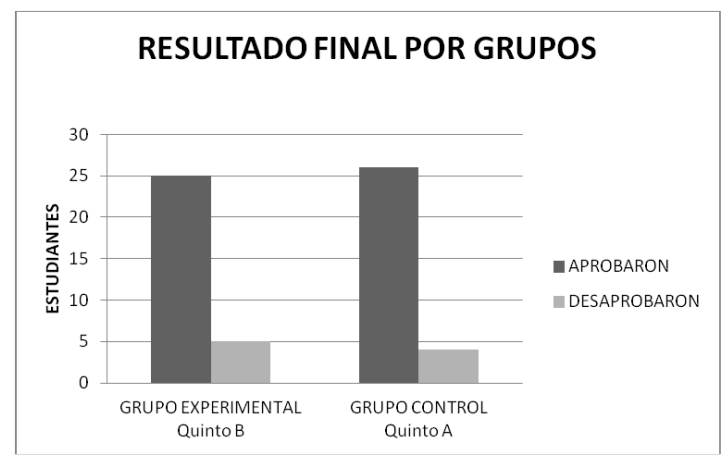

Fig.6. Proyecto final.

Lo anterior indica que hubo un aprendizaje significativo en los estudiantes que utilizaron el programa Scratch como mediación didáctica, reflejado esto en la mejora de su desempeño en las pruebas. Además, al comparar el producto final de los grupos experimental y control, se observa que el uso de las TIC contribuye elocuentemente en la apropiación de conocimientos de ciencias naturales. En este sentido, se puede decir que el ambiente de enseñanza-aprendizaje y la metodología, fueron apropiadas para fortalecer el conocimiento de las temáticas tratadas. 
Los resultados anteriores son consistentes con los hallazgos de Lozano et al. (2016), quienes emplearon Scratch para el desarrollo de habilidades de pensamiento en niños del grado sexto, encontrando que es altamente efectivo comparada con las clases tradicionales. Si bien este trabajo se centró en el área de ciencias naturales, Scratch se puede aplicar a cualquier materia que se desee.

El programa Scratch es una plataforma para programar e interactuar con el computador, es decir, aprender aprendiendo. Este software tiene una interface que permite realizar cualquier tipo de programación, desde la más simple a la más compleja, dependiendo del nivel de programación (Ríos, 2015). En palabras sencillas, programar en Scratch es como jugar con LEGO, ya que provee un sinfín de bloques de colores que nos dan instrucciones para desarrollar cualquier tipo de programación, para interactuar y para armar nuestro propio juego. Este software brinda un entorno emocionante para los niños, porque es colorido, y lo más importante, el estudiante de primaria tiende a estar familiarizado con cosas animadas y eso es lo que genera un ambiente de trabajo motivante.

\section{CONCLUSIONES}

El ambiente de enseñanza debe ajustarse a los requerimientos del entorno, de modo que se apoye al estudiante para que alcance sus metas de aprendizaje. En este sentido, el docente debe ser competitivo en el uso de las herramientas TIC, adaptando los materiales disponibles en la institución educativa para optimizar su integración en el proceso de enseñanza aprendizaje.

La inclusión del Software Scratch en la educación facilita atraer la curiosidad del niño por su interactividad y colorido, lo cual lo ubica entre los mejores programas para iniciar la programación básica. Adicionalmente, Scratch está a la vanguardia de los procesos de enseñanzaaprendizaje que facilitan la comprensión de los temas, estimulando al niño a la construcción de su propio conocimiento.

Al programar en scratch, aparte de aprender los fundamentos de programación, se promueve el cambio pedagógico, generando una mayor motivación en el estudiante, ya que la tecnología es una alternativa didáctica con la que el niño se divierte. Sin embargo, se le debe dar un buen uso desde el punto de vista pedagógico, para que el estudiante pueda alcanzar las metas de aprendizaje, a la vez que se facilita la labor del docente en el aula.

Los hallazgos indican que Scratch puede servir como herramienta integradora de diferentes áreas, como en el caso de las ciencias naturales aquí presentado, pues su uso tecnológico aporta significativamente en el aprendizaje del estudiante. En este sentido, las actividades de aula deben permitir que el estudiante no solamente adquiera las competencias del área específica, sino que también comprenda el uso de la herramienta tecnológica.

\section{REFERENCIAS}

Angarita V, M. A., Fernández M, F. H., y Duarte, J. E. (2014). La didáctica y su relación con el diseño de ambientes de aprendizaje: una mirada desde la enseñanza de la evolución de la tecnología. Revista de Investigación, Desarrollo e Innovación, 5 (1), 46-55. doi:https://doi.org/10.19053/20278306.31 38

Avella I, C. P., Sandoval V, E. M., y Montañez T, C. (2017). Selección de 
herramientas web para la creación de actividades de aprendizaje en Cibermutua. Revista de investigación, Desarrollo e Innovación, 8(1), 107-120. doi:

10.19053/20278306.v8.n1.2017.7372

Barrera M, C. E, Fernández M, F. H., \& Duarte, J. E. (2017) diseño de un ambiente de aprendizaje mediado por TIC para la enseñanza de operadores mecánicos orientado al grado séptimo de la educación básica, en el colegio Boyacá de Duitama. Revista Colombiana de Tecnologías de Avanzada, 2 (30). doi:

https://doi.org/10.24054/16927257.v30.n3 0.2017 .2740

Barrera M, M., Fernández M, F. H., y Duarte, J. E. (2017). Aprendizaje basado en proyectos colaborativos mediados por TIC para el desarrollo de competencias en estadística. Saber, Ciencia y Libertad, 12 (2), 220-232. Recuperado de:http://www.sabercienciaylibertad.org/oj s/index.php/scyl/article/view/247

Berdugo P, D. J., Duarte, J. E., y Fernández M, F. H. (2018). Desarrollo de un ambiente de aprendizaje mediado con TIC para la enseñanza de la educación económica financiera. Revista Colombiana de Tecnologías de Avanzada, 1 (31), 160167.

doi:https://doi.org/10.24054/16927257.v3 1.n31.2018.2778

Buitrago G, S. (2014). Relación entre la convergencia de medios y la experiencia de usuario. Dos iniciativas creativas en Colombia. Revista De Investigación, Desarrollo E Innovación, 4(2), 79-86. doi:http://dx.doi.org/10.19053/20278306.2 958

Cadena M, E., Eslava B, H. J., y Páez P, I. P. (2015). CAPA FISICA Y ALGORITMOS DE PLANIFICACIÓN
DE ENLACE DESCENDENTE EN LTE Y WiMAX. Revista Colombiana de Tecnologías De Avanzada, 2 (26), 28-30.

Cárdenas S, R. N., y Martínez C, D. (2015). El paisaje sonoro, una aproximación teórica desde la semiótica. Revista de Investigación, Desarrollo e Innovación, 5(2), 129-140. doi:https://doi.org/10.19053/20278306.37 17

Chaupart, J. M., Corredor, M V., y Martin, G. (2014). El tutor, el estudiante y su nuevo rol. Guadalajara: desarrollo de ambientes de aprendizaje en educación a distancia. Universidad de Guadalajara.

Recuperado de:https://investigacion.ilce.edu.mx/panel ᄀ_control/doc/Rayon_Parra.pdf.

Chevallard, Y., y Johsua, M. A. (1982). Un exemple d'analyse de la transposition didactique. Lanotion de distance, Recherches en didactique des mathématiques, 3 (2), 157-289. Chiappe, A., y Romero, R. C. (2018). Condiciones para la implementación del m-learning en educación secundaria: un estudio de caso colombiano. Revista mexicana de investigación educativa, 23(77), 459-481. Recuperado de:http://www.scielo.org.mx/scielo.php?sc ript=sci_arttext $\&$ pid=S140566662018000 200459\&lng=es\&tlng=es.

Criollo, B., Alvarado, J., y Numpaque, H. (2014). Control PID de temperatura y dosificasión de ph para la producción de gas metano a partir de la digestión anaeróbica de residuos sólidos orgánicos. Revista Colombiana de Tecnologías de Avanzada, 2 (24), 134-141. doi:https://doi.org/10.24054/16927257.v2 4.n24.2014.2339 
Cruz R, G. A., Molina B. M. A., y Valdiri V. V. (2019). Vigilancia tecnológica para la innovación educativa en el uso de bases de datos y plataformas de gestión de aprendizaje en la universidad del Valle, Colombia. Revista de Investigación, Desarrollo e Innovación, 9 (2). doi:10.19053/20278306.v9.n2.2019.9175

Escamilla, H. (2009). Perspectivas de la Tecnología Educativa: Diseño instruccional unidad didáctica. Recuperado de: http://www.scribd.com/doc/15790655/Pers pectivas-de-la-Tecnologia-Educativa

Galeano B, C. J., Bellón M, D., Zabala V, S. A., Romero R, E., y Duro N, V. (2018). Identificación de los pilares que direccionan a una institución universitaria hacia un Smart-Campus. Revista de Investigación, Desarrollo e Innovación, 9 (1), 127145.doi:https://doi.org/10.19053/2027830 6.v9.n1.2018.8511

García A, R. A., Fernández M, F. H., y Duarte, J. E. (2017). Modelo de integración de las TIC en instituciones educativas con características rurales. Revista Espacios, 38 (50), 26. Recuperado de:http://www.revistaespacios.com/a17v3 8n50/17385026.html

Garrido B, S. (2015). Scratch para niños..y no tan niños. Recuperado de:

http://dedescargar.wmplondon.org.uk/grati s/B00U9PUZI8-scratch-para-ninos-y-notan-ninos.html

Giraldo P, J. E., Ruiz N, M. A., Rosero N, C. A., y Zapata P, L. N. (2017).

Formación en competencias específicas para la industria del software colombiano. Experiencias del uso del aprendizaje basado en proyectos. Revista Colombiana de Tecnologías de Avanzada, 1 (27).
Recuperado

de:http://ojs.unipamplona.edu.co/ojs_vicei nves/index.php/RCTA/article/view/2529/0

Gutiérrez R, C. (2018). Fortalecimiento de las competencias de interpretación y solución de problemas mediante un entorno virtual de aprendizaje. Revista de Investigación, Desarrollo e Innovación, 8(2), 279-293. doi: https://doi.org/10.19053/20278306.v8.n2.2 018.7170

Guzmán L, J., I., y Álvarez, J. F. (2014). Propuesta de un generador de aplicaciones educativas basadas en televisión digital usando arquitectura de computo en la nube. Revista Colombiana de Tecnologías de Avanzada, 2 (24).

Jiménez E, A., y Sánchez B, D. M. (2019). La práctica pedagógica desde las situaciones a-didácticas en matemáticas. Revista de Investigación, Desarrollo e Innovación, 9 (2).

doi:10.19053/20278306.v9.n2.2019.9179

Jiménez P, I., Martelo G, R., y Peña P, M. (2017). Diagnóstico sobre accesibilidad e integración digital dentro del sector universitario colombiano. Saber, Ciencia Y Libertad, 12(1), 225-235. Recuperado de:

http://www.sabercienciaylibertad.org/ojs/i ndex.php/scyl/article/view/227

Lopéz de P, L., Polanco P, V., y Correa C, L. (2017). Mirada a las investigaciones sobre formación investigativa en la universidad latinoamericana: estado del arte 2010 a 2017. Revista de Investigación, Desarrollo e Innovación, 8(1), 77-95. doi:https://doi.org/10.19053/20278306.v8. n1.2017.7371 
Lozano M, P. A., Guerrero C, B. A., y

Gordillo O, W. D. (2016). Scratch y

Makey Makey: herramientas para

fomentar habilidades del pensamiento de

orden superior. Redes de Ingeniería, 7 (1), 16-23. doi:

https://doi.org/10.14483/udistrital.jour.red es.2016.1.a4

Marino V, H., Mendoza, L., y Gualdrón G, O. (2017). Medición automática de variables antropométricas para la evaluación de la respiración usando visión artificial. Revista de Investigación, Desarrollo e Innovación, 8(1), 161-169. doi:https://doi.org/10.19053/20278306.v8. n1.2017.7407

Martínez L, L. G., y Gualdrón P, E. (2018). Fortalecimiento del pensamiento variacional a través de una intervención mediada con TIC en estudiantes de grado noveno. Revista de Investigación, Desarrollo e Innovación, 9 (1), 91-102. doi:

https://doi.org/10.19053/20278306.v9.n1.2 018.8156

Mejía B, D., Peña C, C. A., y Riaño J, C. I. (2017). SISTEMA SOLAR PARA LA OPERACIÓN DE UN ROBOT AGRÍCOLA. Revista Colombiana de Tecnologías de Avanzada, 1 (26).

Recuperado de:

http://revistas.unipamplona.edu.co/ojs_vic einves/index.php/RCTA/article/view/2533

Moreno L, J., Robles, G., y Román G, M. (2015). Análisis automático de proyectos Scratch para evaluar y fomentar el pensamiento computacional. Revista de Educación a Distancia, 46.

Moya E, P. I., Cortés R, N. C., y Martínez C, A. G. (2019). Proceso de toma de decisiones en mipymes hoteleras de Boyacá, Colombia. Revista de
Investigación, Desarrollo e Innovación, 9 (2). doi:

10.19053/20278306.v9.n2.2019.9159

Niño V, J. A., Martínez D, L. Y., Fernández M, F. H. (2016). Mano robótica como alternativa para la enseñanza de conceptos de programación en Arduino. Revista Colombiana de Tecnologías de Avanzada, 2 (28), 132-139.

Niño, J. A., Morán B, R. A., y Fernández M, F. H. (2018). Educación inclusiva: un nuevo reto para la labor docente en el siglo XXI. Infometric@ - Serie Sociales y Humanas, 1 (2), 74-94. Recuperado de: http://cienciometrica.com/infometrica/inde x.php/ssh/article/view/78

Ordóñez O, O., Gualdrón P, E., y Amaya F, G. (2019). Pensamiento variacional mediado con baldosas algebraicas y manipuladores virtuales. Revista de Investigación, Desarrollo e Innovación, 9 (2). doi:

10.19053/20278306.v9.n2.2019.9180

Páez B, E., Corredor C. E., Y Fonseca C, J. (2016). Evaluación del uso de herramientas sincrónicas y asincrónicas en procesos de formación de las ciencias agropecuarias. Ciencia y Agricultura, 13(1), 77-90. doi: https://doi.org/10.19053/01228420.4808

Palma S, C. A., y Sarmiento P, R. E. (2015). Estado del arte sobre experiencias de enseñanza de programación a niños y jóvenes para el mejoramiento de las competencias matemáticas en primaria. Revista mexicana de investigación educativa, 20(65), 607-641. Recuperado de:

http://www.scielo.org.mx/scielo.php?scrip $\mathrm{t}=$ sci_arttext\&pid=S140566662015000200013\&lng=es\&tlng=es. 
Papert, S. (1980). Mindstorms: children, computers and powerful ideas. Basic Books.

Piratoba G, R, P., Y Rojas M, C. E. (2014). Cambios en las concepciones iniciales e inducidas sobre la naturaleza de las matemáticas y su didáctica, en estudiantes de un programa de licenciatura en matemáticas y estadística. Revista de Investigación, Desarrollo e Innovación, 5(1), 32-45.

doi:https://doi.org/10.19053/20278306.31 40

Pulido H, D. C. (2018). Gestión del conocimiento y las tecnologías de la información y la comunicación (tic). Infometric@ - Serie Sociales y Humanas, 1(1). Recuperado de:http://cienciometrica.com/infometrica/i ndex.php/ssh/article/view/3

Resnick, M., Maloney, J., Monroy H, A., Rusk, N., Eastmond, E., Brennan, K., Millner, A., Rosenbaum, E., Siver, J., Silverman, B., y Kafay, Y. (2009).

Scratch: programming for all. Communications of the ACM, 52(1), 6067. doi: 10.1145/1592761.1592779.

Ríos M, G. C. (2015). Scratch + ABP, como estrategia para el desarrollo del pensamiento computacional. Colombia: Universidad EAFIT.

Riscanevo E, L. (2016). La teoría de la práctica social del aprendizaje en la formación de profesores de matemáticas. Revista de Investigación, Desarrollo e Innovación, 7(1), 93-110. doi:https://doi.org/10.19053/20278306.v7. n1.2016.5635

Ruiz M, E., y Duarte, J. E. (2018). Diseño de un material didáctico computarizado para la enseñanza de oscilaciones y ondas, a partir del estilo de aprendizaje de los estudiantes. Revista de Investigación, Desarrollo e Innovación, 8 (2), 295-309. doi:https://doi.org/10.19053/20278306.v8. n2.2018.7966

Salcedo R, R. Y., Fernández M, F. H., \& Duarte, J. E. (2017). Unidad didáctica para la enseñanza de probabilidad mediada por un OVA, orientada a un colegio rural del municipio de Paipa. Revista Colombiana de Tecnologías de Avanzada, 2 (30). doi:https://doi.org/10.24054/16927257.v3 0.n30.2017.2739

Sanz P, R., y González B, A. (2018). La educación sigue siendo un "tesoro". Educación y docentes en los informes internacionales de la UNESCO. Revista iberoamericana de educación superior, 9(25), 157-174. doi:

https://dx.doi.org/10.22201/iisue.2007287 2e.2019.25.34

Sepúlveda D, O. (2015). Estudio del conocimiento didáctico-matemático del profesor universitario: un marco teórico de investigación. Revista de Investigación, Desarrollo e Innovación, 6(1), 29-43. doi: https://doi.org/10.19053/20278306.4048

Serrano, G. (2012). Programación scratch para niños. USA: Createspace.

Pasos T, L. A., Tenorio S, G. C., y Ramírez M, M. S. (2015). Atributos de la innovación en el marco del movimiento educativo abierto para desarrollar competencias matemáticas. Actualidades investigativas en educación, 15(3), 1-24.

Vega M, N. C., y Ruiz R, A. M. (2018). Retos y desafíos de la educación superior para responder a la demanda de profesionales en la subregión del Oriente Antioqueño Colombiano. Revista de Investigación, Desarrollo e Innovación, 9 
(1), 115-126.

doi:https://doi.org/10.19053/20278306.v9.

n1.2018.8510

Vidal, C., Cabezas, C., Parra, J., Y López, L. (2015) experiencias prácticas con el uso del lenguaje de programación scratch para desarrollar el pensamiento algorítmico de estudiantes en chile. Formación

Universitaria 8(4), 23-32. doi:

http://dx.doi.org/10.4067/S071850062015000400004

Zuluaga D, J. F. (2017). Relación entre conocimientos, saberes y valores: un afán por legitimar los saberes más allá de las ciencias. Revista de Investigación,

Desarrollo e Innovación, 8(1), 61-76. doi:

10.19053/20278306.v8.n1.2017.5973 